\title{
Infrared Camera (IRC) onboard ASTRO-F
}

\author{
Takehiko Wada $^{a}$,Naofumi Fujishiro ${ }^{a}$, Daisuke Ishihara ${ }^{b}$, Hirokazu Kataza ${ }^{a}$, Woojung $\operatorname{Kim}^{a}$, \\ Hideo Matsuhara ${ }^{a}$, Toshio Matsumoto $^{a}$, Hiroshi Murakami $^{a}$, Takashi Onaka ${ }^{b}$, \\ Norihide Takeyama ${ }^{b}$, Kazunori Uemizu $^{d}$, Munetaka Ueno ${ }^{e}$, and Hidenori Watarai ${ }^{a}$ \\ ${ }^{a}$ The Institute of Space and Astronautical Science, Sagamihara, Kanagawa, Japan \\ ${ }^{b}$ School of Science, The University of Tokyo, Bunkyo, Tokyo, Japan \\ ${ }^{c}$ Nishi-Harima Astronomical Observatory, Sayo, Hyogo, Japan \\ $d$ School of Arts and Sciences, The University of Tokyo, Meguro, Tokyo, Japan
}

\begin{abstract}
The infrared camera(IRC) onboard ASTRO-F is designed for wide-field imaging and spectroscopic observations at near- and mid-infrared wavelengths. The IRC consists of three channels; NIR, MIR-S and MIR-L, each of which covers wavelengths of 2-5, 5-12 and 12-26 micron, respectively. All channels adopt compact refractive optical designs. Large format array detectors (InSb 512x412 and Si:As IBC 256x256) are employed. Each channel has 10x10 arcmin wide FOV with diffraction-limited angular resolution of the $67 \mathrm{~cm}$ telescope of ASTRO-F at wavelengths over 5 micron. A 6 -position filter wheel is placed at the aperture stop in each channel, and has three band-pass filters, two grisms/prisms and a mask for dark current measurements. The 5 sigma sensitivity of one pointed observation is estimated to be 2,11 and 62 micro-Jy at 4, 9, 20 micron bands, respectively. Because ASTRO-F is a low-earth orbiting satellite, the observing duration of each pointing is limited to 500 seconds. In addition to pointed observations, we plan to perform mid-infrared scanning observation. Fabrications of the flight-model of NIR, MIR-S, and the warm electronics have been mostly completed, while that of MIR-L is underway. The performance evaluation of the IRC in the first end-to-end test (including the satellite system) is presented.
\end{abstract}

Keywords: infrared astronomy, infrared camera, infrared instrumentation, space mission, ASTRO-F

\section{INTRODUCTION}

ASTRO-F $\mathrm{F}^{1,2}$ is the second Japanese infrared astronomical satellite, which is scheduled to be launched with the $\mathrm{M}-\mathrm{V}$ rocket of the Institute of Astronautical Science (ISAS) in early spring of 2004. ASTRO-F is dedicated for the general infrared sky survey with 2-6 order of magnitude better sensitivity than the IRAS survey. The scientific payload consists of a $67-\mathrm{cm}$ cooled telescope ${ }^{3}$ of silicon carbide mirrors and two focal-plane instruments, the Infrared Camera (IRC) ${ }^{4,5}$ and the Far-Infrared Surveyor (FIS). ${ }^{6,7}$ The whole scientific payload is installed in a cryostat $^{8}$ cooled by 170-liter liquid helium and two 2-stage Stirling cycle coolers.

ASTRO-F has a sun-synchronous polar orbit along the twilight zone, which is similar to the IRAS orbit. The spacecraft spins around the sun-pointed axis once an orbit so that the telescope always points at the zenith. The far infrared all-sky survey is performed by the FIS in this scanning mode. The IRC is mainly operated in the pointed mode, in which the spacecraft is fixed in the inertial space and the telescope is pointed at a target position. The pointed observation can be scheduled up to 3 times per orbit. The duration of one pointed observation is limited to approximately 10 minutes in order to prevents the direct earth shine from coming into the cooled baffle of the telescope. In addition, we currently plan to perform mid-infrared all sky survey by the IRC in the scanning mode. Performance evaluation of the focal plane arrays for the IRC all sky survey is shown in Ishihara et al. ${ }^{9}$

The ASTRO-F mission is divided into three phases. Phase 1 is the first 180 days, where the FIS all sky survey has a priority. We plan to perform the IRC all sky survey in this phase. Phase 2 lasts until the liquid

Further author information: e-mail: wada@ir.isas.ac.jp 

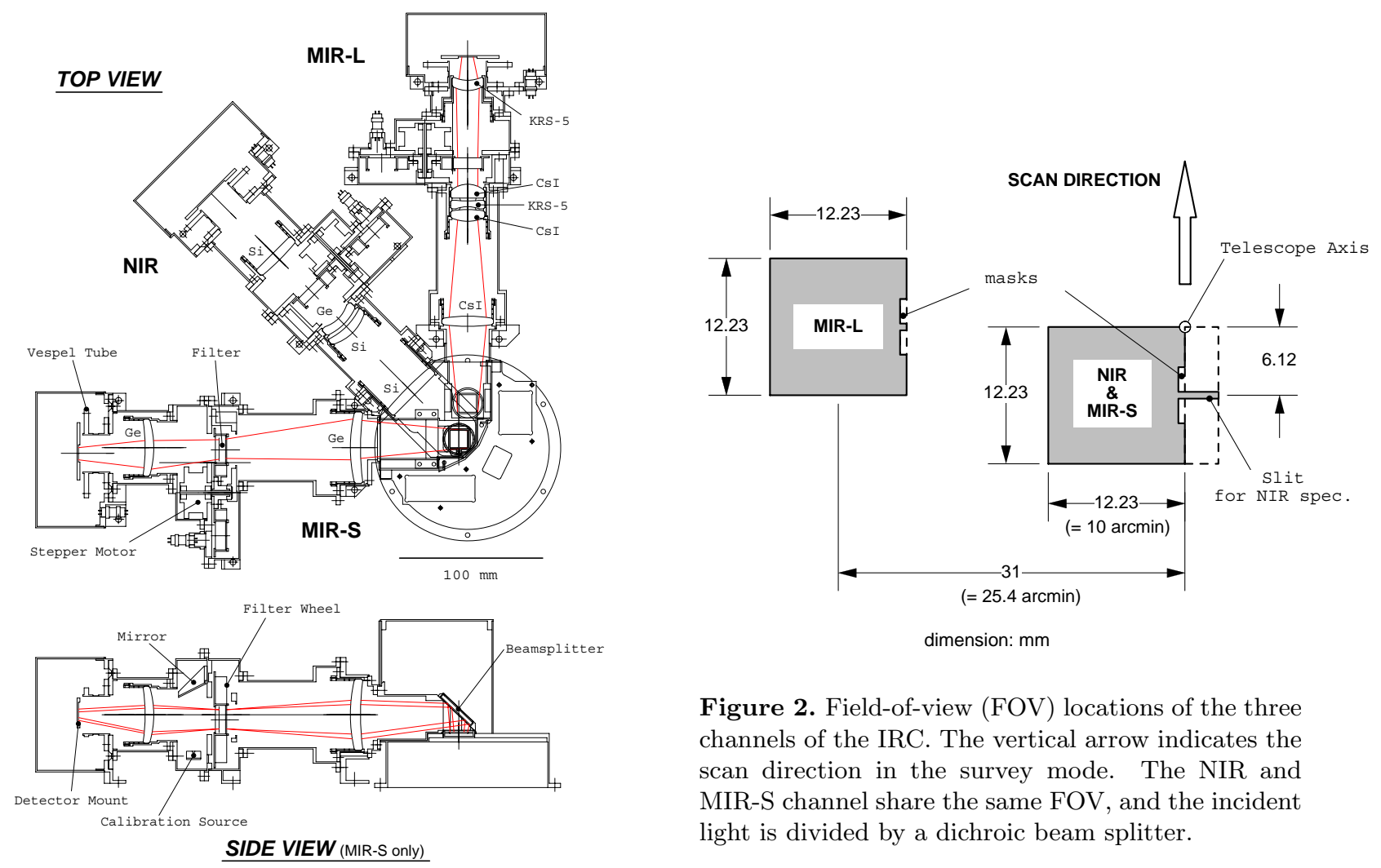

Figure 2. Field-of-view (FOV) locations of the three channels of the IRC. The vertical arrow indicates the scan direction in the survey mode. The NIR and MIR-S channel share the same FOV, and the incident light is divided by a dichroic beam splitter.

Figure 1. A top view of the IRC (top) and a side view of the MIR-S (bottom). The optical design for each channel is indicated together with the lens material.

helium is exhausted, where the IRC pointed observation has a priority. The duration of phase 2 is estimated to be 300 days. Phase 3 starts after the liquid helium runs out, where only the near-infrared channel of the IRC can operate. The duration of phase 3 depends on the lifetime of the Stirling cycle mechanical coolers. We expect to have 1500 and 5900 pointed observations for the IRC in phase 1 and 2, respectively.

A major scientific objective of the IRC is the investigation of the process of birth and evolution of galaxies by means of deep photometric and spectroscopic surveys in near- and mid-infrared wavelengths for a wide area of the sky. In this paper, we describe the design and the current development status of the IRC.

\section{OPTICAL AND MECHANICAL DESIGN}

The IRC consists of three channels: NIR, MIR-S, and MIR-L. Figure 1 shows the design of the IRC and Figure 2 shows the location of the field-of-view of each channel on the focal plane of the telescope. Refractive system was adopted for the camera optics for compactness. A 6-position filter wheel is installed at the aperture stop of each channel. The focal plane of each channel is equipped with a large format detector array : $512 \times 412 \mathrm{InSb}$ array for the NIR and $256 \times 256 \mathrm{Si}$ :As array for the MIR-S and MIR-L channels. The specifications of the IRC optical design are summarized in Table 1.

The NIR channel consists of four lenses ( $\mathrm{Si}-\mathrm{Si}-\mathrm{Ge}-\mathrm{Si}$ ). The Ge lens has aspherical surface figure. Six positions of the filter wheel are allocated to three bandpass filters of Ge substrates, a prism $(\mathrm{CaF})$, a grism $(\mathrm{Ge})$, and a blank mask. The $412 \times 412$ portion of the InSb array corresponds to a $10^{\prime} \times 10^{\prime}$ field on the sky and is used for the imaging and the slit-less spectroscopy. The rest $412 \times 100$ portion has a slit mask for the spectroscopic 
Table 1. Specifications of the IRC Optics.

\begin{tabular}{|c|c|c|c|c|c|}
\hline Channel & Wavelength Range & Filters/Dispersers & $\begin{array}{c}\text { FOV } \\
(\mu \mathrm{m})\end{array}$ & $\begin{array}{c}\left.\text { Plate Scale }^{2}\right) \\
\left(\operatorname{arcsec~pixel~}^{1}\right)\end{array}$ & efficiency $_{(\%)}^{\dagger}$ \\
\hline NIR & $2.0-5.0$ & N2, N3, N4, NG, NP & $10 \times 10$ & 1.46 & 60 \\
\hline MIR-S & $5.0-12$ & S7, S9W, S11, SG1, SG2 & $10 \times 10$ & 2.34 & 40 \\
\hline MIR-L & $12-26$ & L15, L20W, L24, LG1, LG2 & $10 \times 10$ & 2.34 & 30 \\
\hline
\end{tabular}

$\dagger$ development goal

\begin{tabular}{|c|c|c|c||c|c|c||c|c|c|}
\hline Filters & N2 & N3 & N4 & S7 & S9W & S11 & L15 & L20W & L24 \\
\hline Wavelength & $1.8-2.7$ & $2.7-3.7$ & $3.7-5.0$ & $5.5-8.5$ & $6.0-11.5$ & $8.5-13.0$ & $12.5-18.0$ & $14.0-26.0$ & $22.0-26.0$ \\
\hline
\end{tabular}

\begin{tabular}{|c|c|c||c|c||c|c|}
\hline Dispersers & NP & NG & SG1 & SG2 & LG1 & LG2 \\
\hline Type & prism & grism & grism & grism & grism & grism \\
\hline Wavelength & $2.0-5.0$ & $2.5-5.0$ & $5.0-8.0$ & $7.0-12.0$ & $11.0-19.0$ & $18.0-26.0$ \\
\hline Effective R & 30 & 180 & $40-60$ & $40-60$ & 40 & 40 \\
\hline
\end{tabular}

observations of extended sources. The laboratory evaluation of the NIR optics are shown by Kim et al. ${ }^{10}$ The MIR-S channel consists of two Ge lenses with aspherical surface figures. The band-pass filters are made on ZnS substrates. The MIR-L channel consists of five lenses (CsI - CsI - KRS-5 - CsI - KRS-5). The filters are made on CdTe substrates. Grisms for MIR-S and MIR-S are made of KRS-5. Each of the MIR-S and the MIR-L channels have a small slit mask for spectroscopic observations of extended sources. Ultra-wide band-pass filters $(\lambda / \Delta \lambda \sim$ 1.5) are employed for MIR-S and MIR-L channels (S9W and L20W), which enable the highest sensitivity in deep surveys.

Each channel has its own housing made of A6061-T653 aluminum alloy. All the three channels are joined together to a cylindrical structure, which is located at the center of the focal plane. This structure ensures the reproducibility of the alignment. Each channel weighs less than $1.2 \mathrm{~kg}$ and the total weight of the IRC is estimated to be $3.5 \mathrm{~kg}$. A filter wheel, a stepper motor, and a calibration light source are attached to the base plate close to the aperture stop of each channel. The stepper motor is specially designed by Sumitomo Heavy Industry Ltd. for the low-temperature use with low heat dissipation $(<10 \mathrm{~mW} @ 6 \mathrm{~K})$. The filter wheel is directly driven by the stepper motor. The accuracy and its reproducibility of the stepping position are confirmed to be better than $30^{\prime}$ by measurements with an auto-collimator. In order to define the home position of the filter wheel, a pair of photodiode and LED is installed on the wheel housing structure, which detects a hole placed on the wheel. The calibration light source is provided by the NASA Goddard Space Flight Center. The light is reflected by the roughened surface of the blank mask on the filter wheel and illuminates the detector uniformly. This calibration light source is used to monitor the short time scale variations and the pixel-to-pixel variations of the detector response. The absolute calibration and the long term variation is determined by measurements of standard stars in the sky, and the flat-fielding is made by observing the sky background in orbit. The dark image with calibration light source taken in the end-to-end test are shown in Figure 8 and 11.

The focal plane arrays are mounted on custom-made chip-carriers, which are installed on a fanout board. The fanout board is isolated thermally from the housing structure by tubes made of Vespel polyimide.

\section{FOCAL PLANE ARRAYS}

To maximize the advantages of low back ground condition achieved by the cooled space telescope, the detectors must have a high performance in the quantum efficiency, the dark current, and the noise characteristics. Low power operation is also important for the cryogenics.

State-of-the-art large format infrared arrays, a $512 \times 412 \mathrm{InSb}$ array ${ }^{11}$ and two $256 \times 256 \mathrm{Si}$ :As impurity band conduction (IBC) arrays, ${ }^{12}$ manufactured by Raytheon/IRO, are employed for the IRC. Each of the InSb and the Si:As IBC detectors are hybridized by indium bumps to a Si Read-Out Integrated Circuit (ROIC), SBRC-189 and CRC-744, ${ }^{13}$ respectively. Our laboratory tests ${ }^{9}$ of the arrays have demonstrated the good performance of these arrays at cryogenic temperatures, however, it also shows the output offset of the ROIC, 


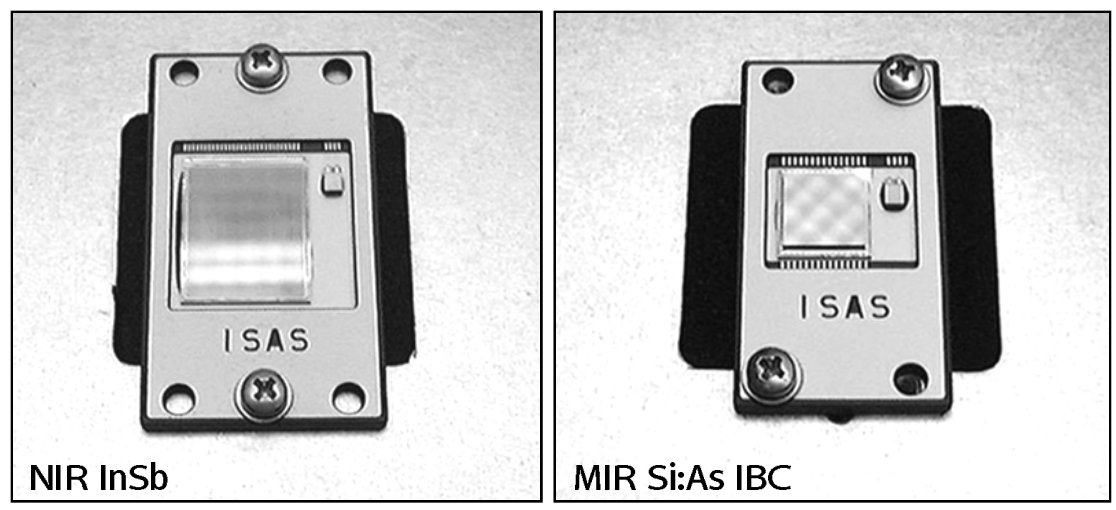

Figure 3. Flight arrays delivered by Raytheon/IRO. The detector arrays are mounted on custom-made chip-carriers with temperature sensors. The array active areas of $\mathrm{InSb}$ and Si:As are $15.4 \times 12.4 \mathrm{~mm}$ and $7.7 \times 7.7 \mathrm{~mm}$, respectively.

the photo-response of the InSb and the dark current of the Si:As change dramatically with the temperatures for $4-10 \mathrm{~K}$.

The ROICs are fabricated by the cryo-CMOS technology and have $30 \mu \mathrm{m}$ pitch unit cells, each of which consists of three P-ch MOS-FETs. The switched source-follower-per-detector design is adopted for the nondestructive read-out of the pixel voltage. The signals from the unit cells are multiplexed into four outputs by a combination of fast (column) and slow (raw) scanning multiplexers. 13 biases including detector bias and 8 clocks are needed to operate a CRC-744 ROIC with Si:As detector. The SBRC-189 is a newly designed ROIC based on the CRC-744 technology with several improvements. One bias line to the unit cell is eliminated in order to simplify the structure. The total number of biases are reduced to 11 for the InSb array. The SBRC-189 $\mathrm{InSb}$ is currently the largest format near-infrared detector array that works for space astronomy.

All the flight detector arrays including the backup arrays have been mounted on the chip-carriers specially designed for the IRC (Figure 3). The chip carriers are made of ceramic $\left(96 \% \mathrm{Al}_{2} \mathrm{O}_{3}\right)$ and the arrays are glued onto the chip-carriers by epoxy resin. Cryogenic temperature sensors (Lake shore Cryotronics Inc., Cernox sensor) are mounted together with the arrays to monitor the temperature accurately. The chip-carrier is attached to the fanout board, a glass-epoxy printed circuit, providing the electrical interface to the outside. The decoupling capacitors for all the bias supply lines are also mounted on the fanout board. The adjustment of the temperatures of the arrays in appropriate ranges ( $6 \mathrm{~K}$ for Si:As and $15 \mathrm{~K}$ for $\mathrm{InSb}$ ) is made by heaters placed next to the chip-carrier.

The control signal for the three arrays are generated by one sequencer in order to avoid the asynchronous mutual-interference. We employ Fowler sampling technique ${ }^{14}$ to read out the detector signal. The arrays are continuously read out in the non-destructive mode even in the integration and the reset, in order to stabilize the array temperature. The change of array temperature causes the change of the offset of the ROIC and prevent us from measuring the correct detector signal.

\section{ELECTRONICS}

The IRC warm electronics (IRC-E) consists of five boards, the CPU, sequencer, peripheral control, array driver, and DC-DC converter boards. The block diagram of the IRC-E is shown in Figure 4. The CPU board is the interface between the satellite system and the IRC. The CPU board receives commands from the data handling unit (DHU) and send back the house keep (HK) data, which are accumulated from the peripheral control board. The peripheral control board drives calibration light sources, filter wheels, heaters, thermometers, focusing mechanism, and provides control signals to the array driver board according to the CPU board.

The sequencer board generates all control sequences for the array operations according to the starting command from the CPU board. The sequencer board consists of sequence memory, frame memory, accumulator, 


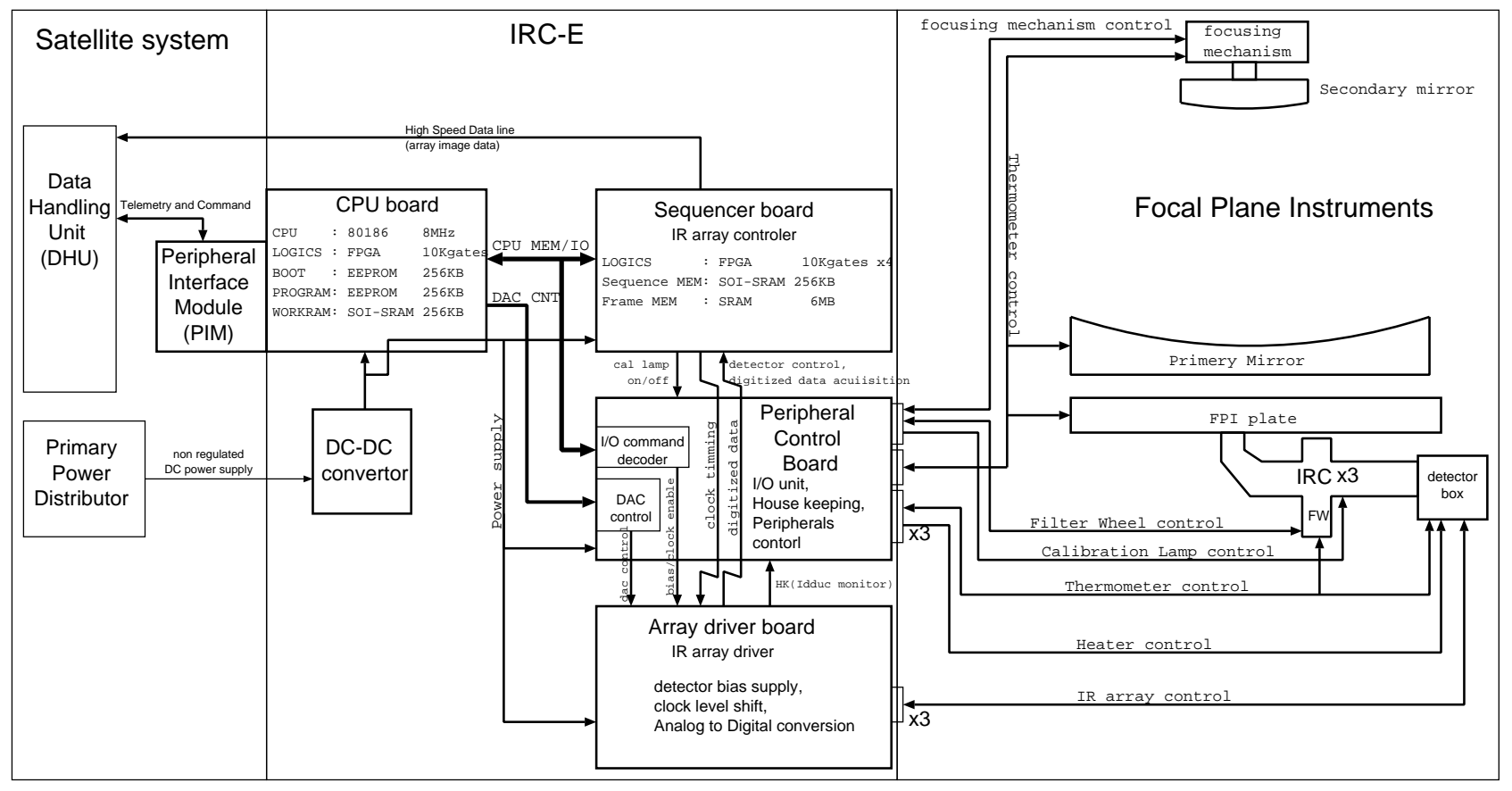

Figure 4. Block diagram of the IRC electronics.

and control logics. Up to 256 independent sequences can be stored. The digital data from the array driver board are accumulated into the frame memory. The accumulator has capability of add/subtract operations between the data from the array driver board and that of the frame memory, which enables correlated double sampling operation and the Fowler sampling.

The array driver board provides bias/clocks for the arrays and digitizes the array output. The high/low states of the clocks from the sequencer board are converted to the actual voltage. The array outputs are digitized by 100kSPS 16bits Analog-to-Digital Converters (ADCs) (Burr-Brown Corporation, ADS7809) together with instrumentation amplifiers (Analog Devices, Inc., AD620). One ADC per output is used in order to increase the total throughput. Totally twelve ADCs for NIR, MIR-S and MIR-L work simultaneously with a common starting signal from the sequencer board in order to avoid mutual interference. In order to reduce digital noise from the CPU board, the CPU bus is eliminated from the array driver board.

In-orbit optimization of the array operation is possible. The clocks are programmed in SRAM sequence memory, which can be reconfigurable. The bias/clock voltage are controlled by 8-bit Digital-to-Analog converters (Analog Devices, Inc., DAC8800) on the array driver board.

The IRC-E box is mounted on the outer shell of the ASTRO-F cryostat. The total ionization dose by the cosmic rays inside the box is estimated to be $7.5 \mathrm{krad}$ in 3 years at most. The selection of the flight electronics parts is made based on the radiation-effect tests. Most of logics are in one-time configurable Field Programmable Gate Arrays (Actel Corporation, A14100A MEC), which have high reliability and good radiation tolerance $(\sim 10$ krad). Memory data upset of the CPU and sequencer board makes the system out of control and may damage the arrays. In order to reduce the single event upset by the cosmic rays, silicon on insulator (SOI) SRAMs, which are free from upset, are used as main memory of CPU and sequencer board. Radiation shielding for DAC8800 is under consideration because it shows poor radiation tolerance $(\sim 2 \mathrm{krad})$.

\section{ESTIMATED SENSITIVITIES}

The estimated sensitivity of the IRC photometric observations for one pointed observation (500 seconds) are shown in Table 2. The back ground level adopted in the calculations and the confusion limits are also shown in 
Table 2. Estimated sensitivity of the IRC

\begin{tabular}{|c||c|c|c|c||c|c|}
\hline \multicolumn{1}{|c||}{ Filter } & \multicolumn{3}{c||}{ Detection limit } & BG level & \multicolumn{2}{c|}{ Confusion Limit $^{a}$} \\
\hline \multicolumn{1}{|c||}{} & \multicolumn{3}{c|}{$(\mu \mathrm{Jy}, 5 \sigma, 500 \mathrm{sec})$} & \multicolumn{2}{c|}{$(\mu \mathrm{Jy})$} \\
\hline & Total & detector limit & BG limit & & LVOL model & ULIRG model \\
\hline N2 & 0.92 & 0.82 & 0.42 & $9.84 \mathrm{e}-08$ & \\
\hline N3 & 1.1 & 1 & 0.34 & $3.69 \mathrm{e}-08$ & \multicolumn{2}{c|}{1} \\
\hline N4 & 1.5 & 1.4 & 0.52 & $3.50 \mathrm{e}-08$ & 3 \\
\hline S7 & 7.1 & 3.1 & 6.4 & $6.63 \mathrm{e}-07$ & 2.7 & 3.3 \\
\hline S9W & 11 & 2.6 & 11 & $1.51 \mathrm{e}-06$ & 5.2 & 17.2 \\
\hline S11 & 24 & 4.8 & 23 & $2.37 \mathrm{e}-06$ & 10.8 & 76 \\
\hline L15 & 56 & 11 & 55 & $3.09 \mathrm{e}-06$ & 30 & 158 \\
\hline L20W & 62 & 8.4 & 61 & $2.74 \mathrm{e}-06$ & 83 & 363 \\
\hline L24 & 140 & 36 & 140 & $2.25 \mathrm{e}-06$ & 149 & \\
\hline
\end{tabular}

a The confusion limits in N3 and N4 bands are estimated based on the model by Franceschini et al. ${ }^{15}$ The confusion limits in the mid-infrared bands are calculated by Pearson et al. ${ }^{16}$ for two evolutionary models.

the same table.

In calculation of the detection limit, we adopt optical efficiency of $60 \%, 40 \%$ and $30 \%$ for NIR, MIR-S and MIR-L channel, respectively. We also adopt the same noise performance in the end-to-end testing (shown in Table 3) can be achieved in case of integration time of 60 and 10 seconds for NIR and MIR channel.

Confusion limit in the near infrared region are estimated based on the model by Franceschini et al, ${ }^{15}$ while that in the mid-infrared bands are calculated by Pearson et al. ${ }^{16}$

Further improvement in NIR channel can be achieved if detector noise is reduced by multi sampling, while further improvement is not expected in the MIR-S and MIR-L channel because it is already limited by the back ground photon noise.

\section{CURRENT STATUS}

The fabrication of the IRC flight model are almost completed and the first end-to-end test including the satellite system has been finished. The scientific instruments will be taken out of the cryostat in September. After some refurbishment, the IRC will be finally installed into the cryostat by the end of 2002 .

\subsection{Development Status}

The development of warm electronics and the CPU software is almost finished. The clock pattern for array control is fixed for imaging mode. We are now developing the clock pattern for scanning mode. Flight model detectors for all channels have been tested and show excellent performance. The development of NIR and MIR$\mathrm{S}$ channel is in the final stage. Most of optical parts for MIR-L channel have been delivered and the optical adjustment starts, while the fabrication of L20W and L24 filters is underway. Figure 5 shows the IRC camera structure and the filter wheel assembly of NIR channel.

\subsection{The First End-to-end Test}

The first end-to-end test of the satellite system were performed from April to July 2002. The test included all aspect of electrical functional checkout of the satellite system in the flight configuration. All satellite components including the cryostat were installed on the space craft and the functional test was performed through the satellite command/telemetry system.

The functional tests of focal-plane instruments were performed from June 23 to June 272002 . The main target for the IRC is to evaluate the performance (especially noise performance) in the flight configuration. 
Table 3. Performance of Detector Arrays.

\begin{tabular}{|c|c|c||c|c|}
\hline & InSb 512 $\times 412(49391)$ & Si:As 256 $\times 256$ IBC $(30164)$ \\
\hline Array Parameter & Requirement & Performance & Requirement & Performance \\
\hline Read Noise $\left(\mathrm{e}^{-}\right)$ & $<20$ & $30.6^{a}$ & $<40$ & $14.2^{b}$ \\
\hline Dark Current $\left(\mathrm{e}^{-} / \mathrm{s}\right)$ & $<10$ & 0.2 & $<100$ & 26 \\
\hline Operability $(\%)$ & $\sim 100$ & 99.9 & $\sim 100$ & 100 \\
\hline Quantum Efficiency $(\%)^{c}$ & 80 & 94 & 40 & 51 \\
\hline
\end{tabular}

${ }^{a}$ Fowler 1 sample, 49 seconds integration.

${ }^{b}$ Fowler 4 sample, 4.6 seconds integration.

${ }^{c}$ Measurements by Raytheon/IRO.

We adopt a conversion factor of 2.78 [microvolt/electron] for SBRC189/InSb and 4.63[microvolt/electron] for CRC744/Si:As. ${ }^{9}$ The system gain of the IRC warm electronics is 29.8 [microvolt/ADU].

The IRC worked without any serious problems. The filter wheel worked perfectly and the secondary mirror adjustment mechanism worked correctly. The heaters and temperature sensors also worked fine. The arrays worked correctly.

We evaluate the performance of the IRC, the point spread function (PSF), the noise, the dark current and the operability of the arrays, in the flight configuration by the data taken in this end-to-end testing. The NIR and MIR-S channel are evaluated, while the MIR-L channel is not evaluated because the PM instead of the FM was installed at that time.

The FWHM of the PSF of the NIR and MIR-S channel (not include the telescope) is estimated to be 2 pixels using the images of pin-holes installed at the focal plane. The dark current and the read out noise of the arrays are evaluated by the images taken with setting the filter wheel at the mask position and the results are summarized in Table 3. The images and the histograms of dark current are shown in Figure 6 and 9 , while the maps and the histograms of the noise are shown in Figure 10 and 7.

In order to check the uniformity of the illumination by the calibration light source and the operability of the arrays, images were taken with setting the filter wheel at the mask position and turning the calibration light source on. The images of the illumination of the calibration light source and the maps of the dead pixels are shown in Figure 8 and 11. The arrays show excellent operability. Especially, the MIR-S array has NO dead pixel.

No significant interference from the satellite system to the IRC were seen in the end-to-end testing. Small excess noises were seen when the FIS warm electronics and the mechanical cooler were working. There were significant interference to the FIS from the IRC. The noise level of the FIS detector when the IRC warm electronics worked was one and a half times as much when the IRC warm electronics did not works. The cause of the interference is under investigation.

\section{ACKNOWLEDGMENTS}

The ASTRO-F project is managed and operated by the Institute of Space and Astronautical Science (ISAS) Japan in collaboration with the groups in universities and institutes in Japan. We are grateful for all the members of the ASTRO-F project for their efforts and help. We also wish to thank D. Jennings for providing calibration source to us.

\section{REFERENCES}

1. H. Murakami, "Current Status of ASTRO-F," ISAS Science Report SP 14, pp. 267-272, 2000.

2. H. Shibai, "ASTRO-F Mission," in IR Space Telescopes and Instruments, Proc. SPIE 4850, 2002.

3. H. Kaneda, T. Onaka, R. Yamashiro, and T. Nakagawa, "Optical Performance of the ASTRO-F Telescope at Cryogenic Temperatures," in IR Space Telescopes and Instruments, Proc. SPIE 4850, 2002. 
4. T. Onaka, et al., "Infrared Camera (IRC) onboard ASTRO-F (IRIS)," ISAS Science Report SP 14, pp. 281288,2000 .

5. H. Watarai, et al., "Infrared camera (IRC) on board ASTRO-F (IRIS): design overview and current status," in UV, Optical, and IR Space Telescopes and Instruments, J. B. Breckinridge and P. Jakobsen, ed., Proc. SPIE 4013, pp. 59-68, 2000.

6. M. Kawada, "FIS - Far-Infrared Surveyor onboard the ASTRO-F," ISAS Science Report SP 14, pp. 273$280,2000$.

7. H. Takahasi, et al., "FIS: far-infrared surveyor on board ASTRO-F (IRIS)," in UV, Optical, and IR Space Telescopes and Instruments, J. B. Breckinridge and P. Jakobsen, ed., Proc. SPIE 4013, pp. 47-58, 2000.

8. T. Nakagawa et al., "Cryogenis System of the Infrared Astronomy Mission ASTRO-F," in IR Space Telescopes and Instruments, Proc. SPIE 4850, 2002.

9. D. Ishihara, et al., "Evaluation of the mid- and near-infrared focal plane arrays for Japanese infrared astronomical satellite ASTRO-F," in IR Space Telescopes and Instruments, Proc. SPIE 4850, 2002.

10. W. Kim, et al., "Imaging Performance of Near Infrared(NIR) channel in Infrared Camera(IRC)on board ASTRO-F," in IR Space Telescopes and Instruments, Proc. SPIE 4850, 2002.

11. P. J. Love, K. J. Ando, J. D. Garnett, N. A. Lum, J. P. Rosbeck, M. S. Smith, and K. P. Sparkman, "Infrared Detectors for Ground-Based and Space-Based Astronomy Applications," in Optical and IR Telescope Instrumentation and Detectors, M. Iye and A. F. Moorwood, ed., Proc. SPIE 4008, pp. 1254-1267, 2000.

12. A. D. Estrada, et al., "Si:As IBC IR Focal Plane Arrays for Ground-Based and Space-Based Astronomy," in Infrared Astronomical Instrumentation, A. M. Fowler, ed., Proc. SPIE 3354, pp. 99-108, 1998.

13. J. Wu, W. J. Forrest, J. L. Pipher, N. Lum, and A. Hoffman, "Development of infrared focal plane arrays for space," Rev. Sci. Instrum. 68, pp. 3566-3578, 1997.

14. A. M. Fowler and I. Gatley "Demonstration of an algorithm for read-noise reduction in infrared arrays," ApJ. 353, pp. L33-L34, 1990.

15. A. Franceschini, L. Toffolatti, L. Toffolatti, P. Mazzei, L. Danese, and G. de Zotti, "Galaxy counts and contribution to the background radiation from 1 micron to 1000 microns," Astron. \& Astrophys. Suppl. 89, pp. 285-310, 1991 .

16. C. P. Pearson, H. Matsuhara, T. Onaka, H. Watarai, T Matsumoto, "ASTRO-F: the next generation of mid-infrared surveys," MNRAS 324, pp. 999-1014, 2001. 

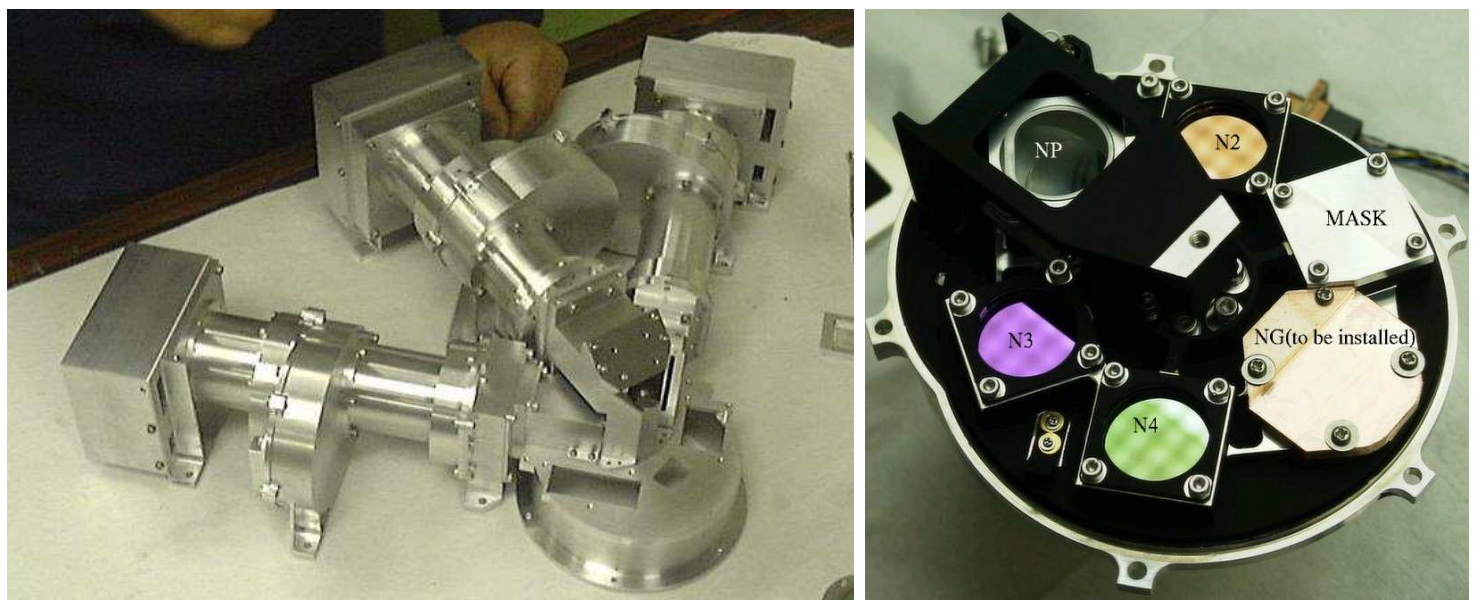

Figure 5. Photographs of the prototype model of the IRC camera structure (left) and the flight model of the NIR filter wheel (right).
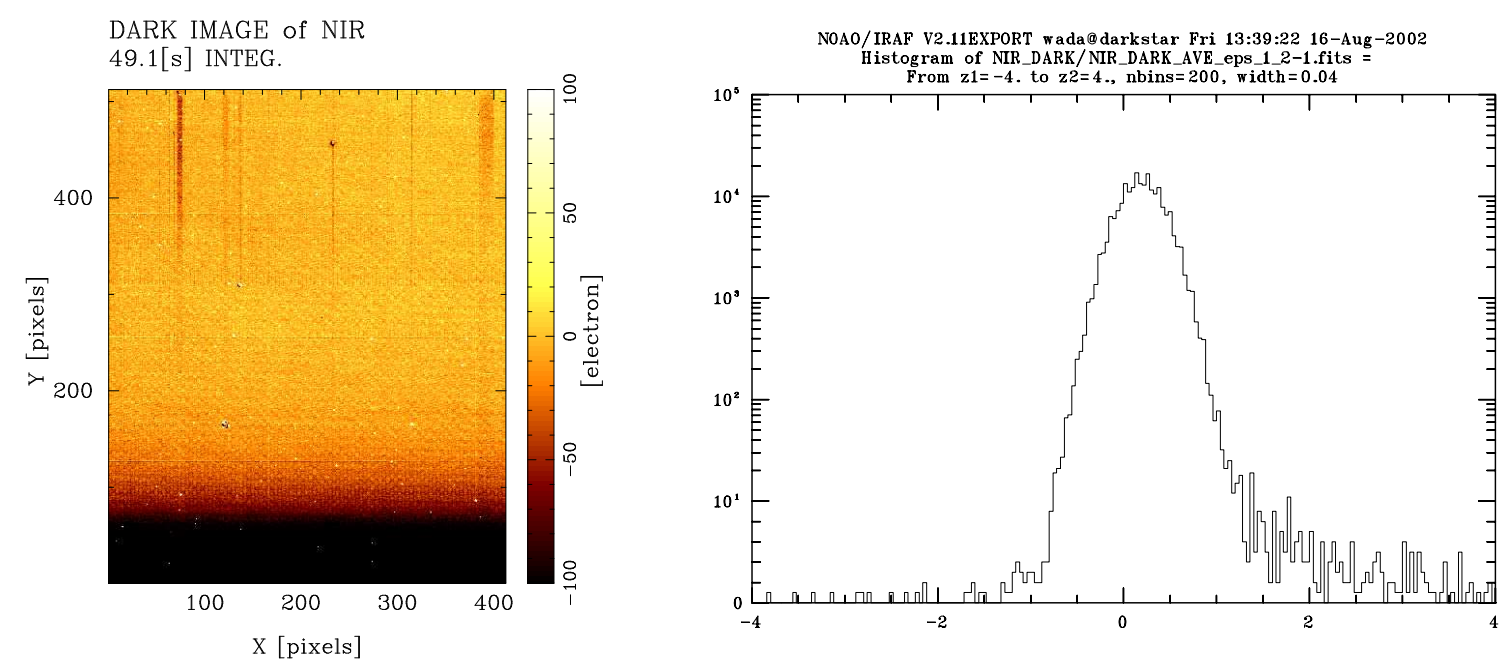

Figure 6. Dark (+offset) image (left) and the histogram (right) of dark current of NIR array. The horizontal axis of the histogram represents dark current $[\mathrm{e} / \mathrm{s}]$. Dark current is estimate from two dark images, which have different integration time (49.0 and 4.7 second) in order to cancel the offset. 

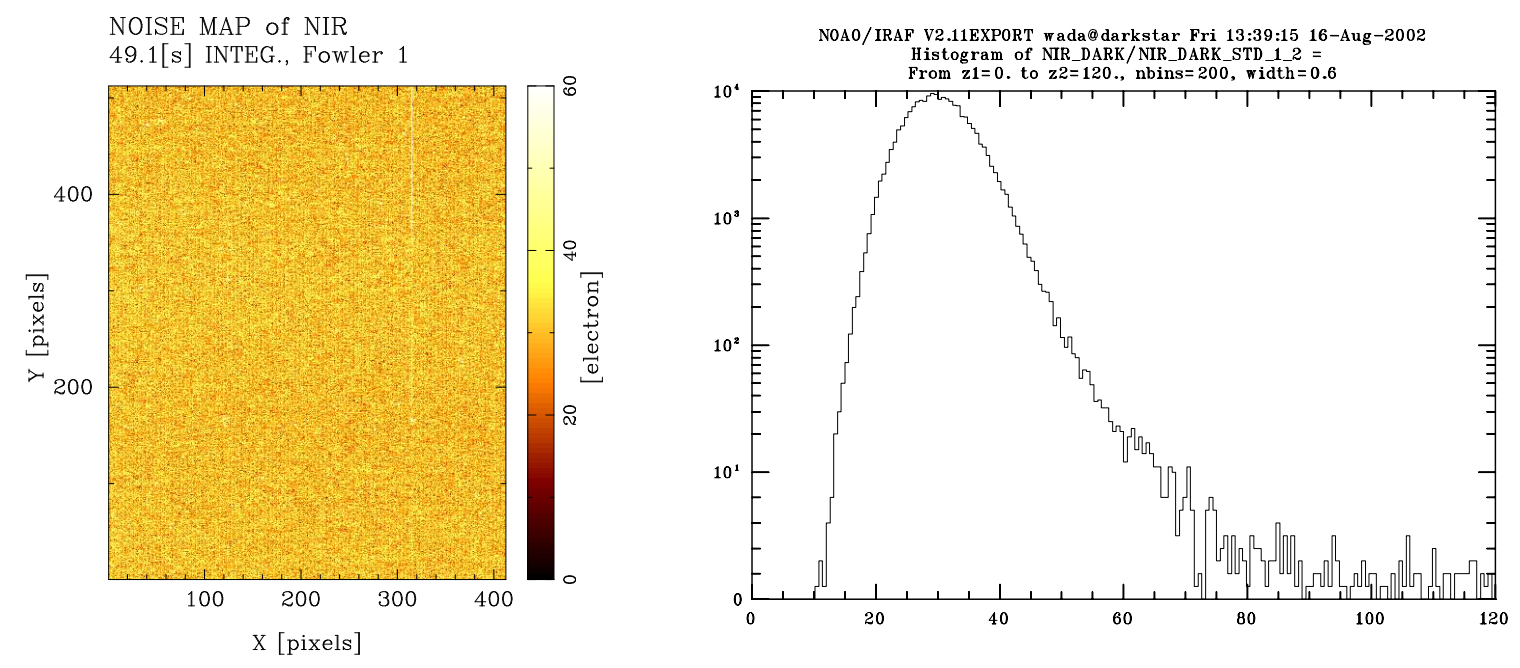

Figure 7. The Map (left) and histogram (right) of noise of NIR array. The horizontal axis of the histogram represents read out noise [e] including IRC warm electronics system noise. The noise is estimated by the standard deviation of each pixel value among the twelve frames in a sequence.

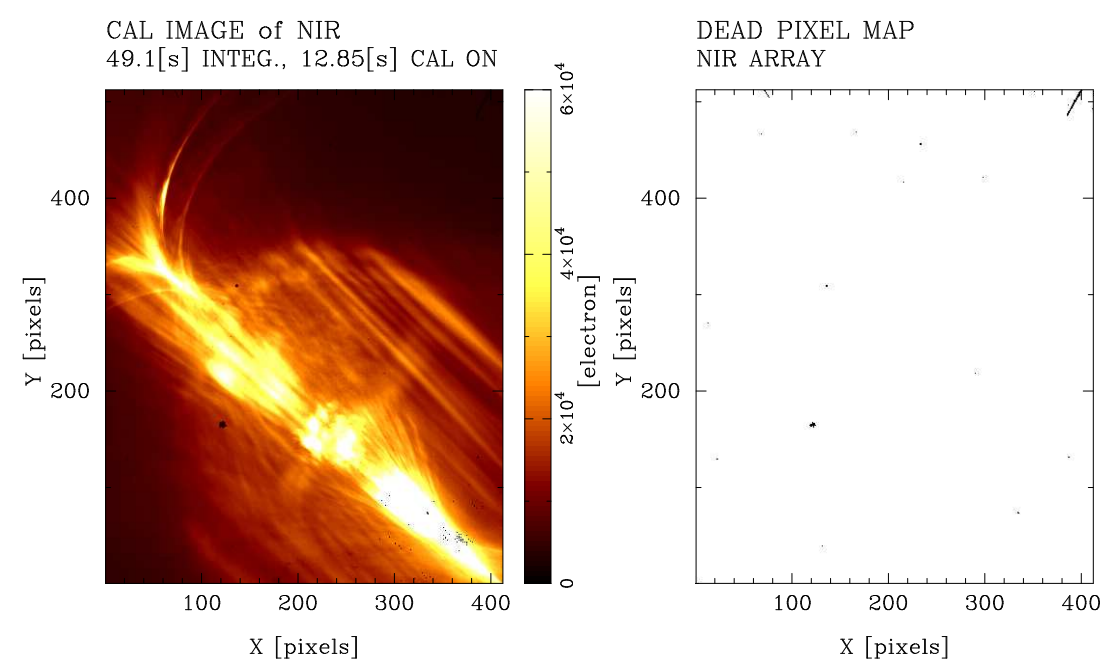

Figure 8. NIR image of calibration lamp (left) and bad pixel map (right) in NIR array. Dark (and offset) are subtracted from the lamp image. The pixels whose photo response is less than 500 electron are shown as dead pixels. 
DARK IMAGE of MIRS

$4.7[\mathrm{~s}]$ INTEG.

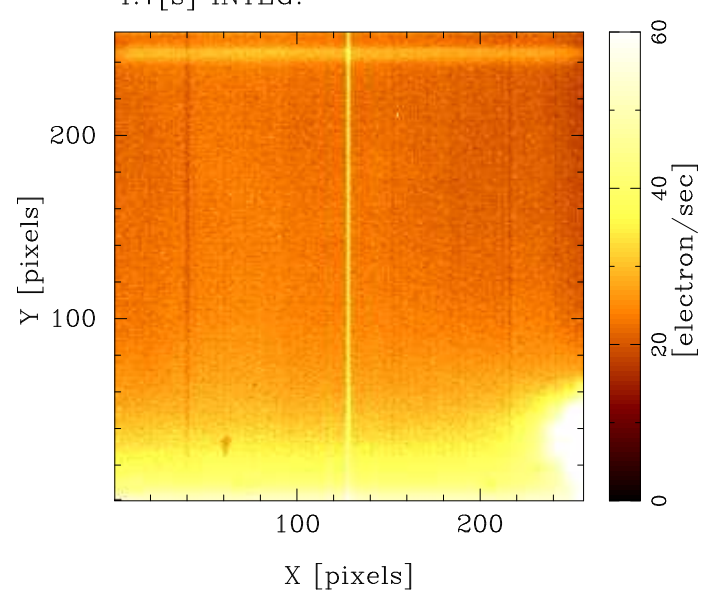

NOA0/IRAF V2.11EXPORT wada@darkstar Fri 13:41:15 16-Aug-2002 From $\mathrm{z} 1=0$, to $\mathrm{z} 2=120$, nbins $=200$, width $=0,6$

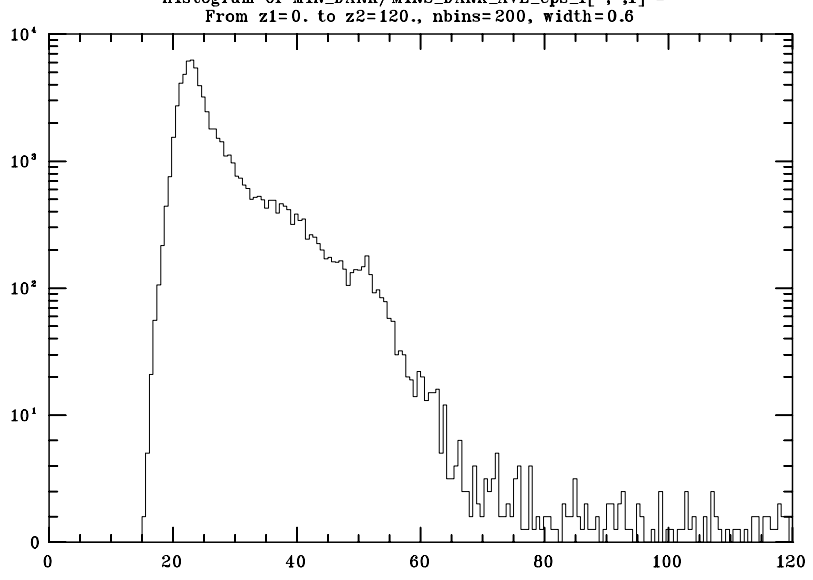

Figure 9. Dark (+offset) image (left) and the histogram (right) of dark current of MIR-S array. The bright spot at the lower right conner is due to the emission from the output FET of the ROIC. The horizontal axis of the histogram represents dark current $[\mathrm{e} / \mathrm{s}]$.
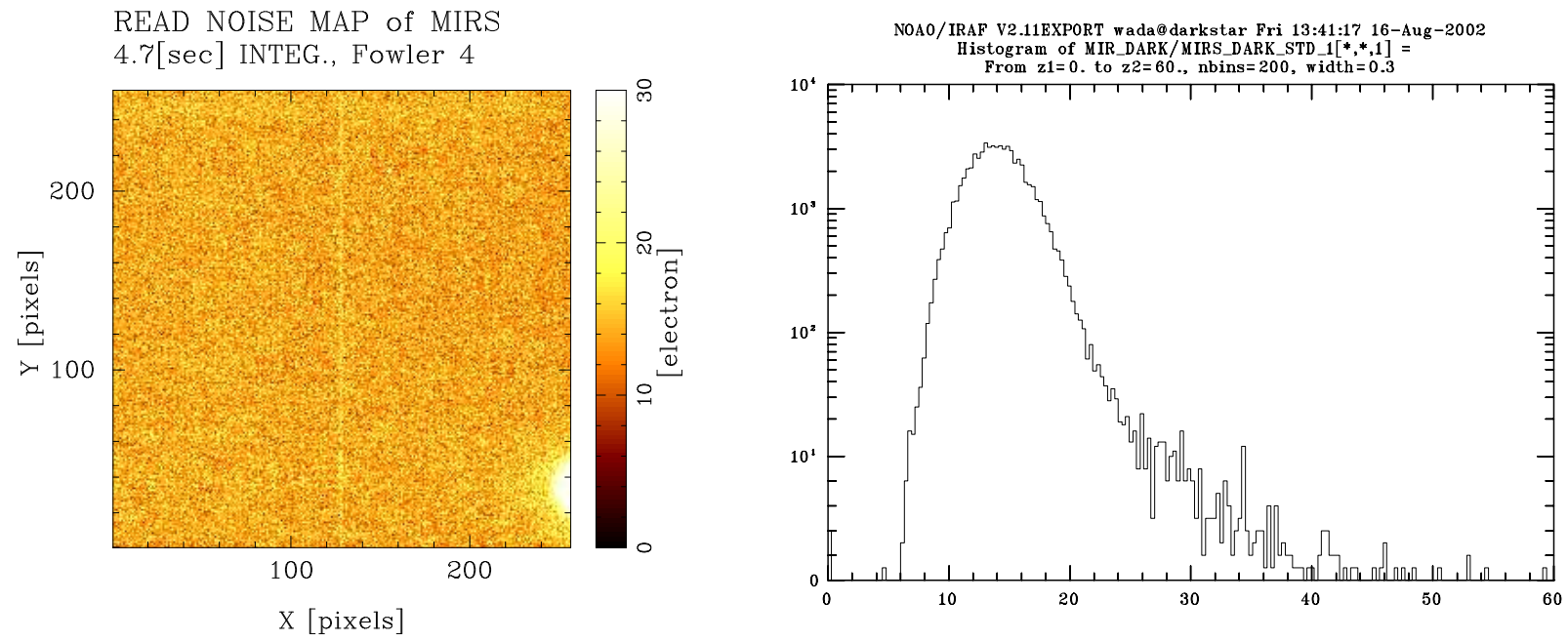

Figure 10. The Map (left) and histogram (right) of noise of MIR-S array. The horizontal axis of the histogram are read out noise [e] including IRC warm electronics system noise. The noise is estimated by the standard deviation of each pixel value among the twelve frames taken in a sequence. 


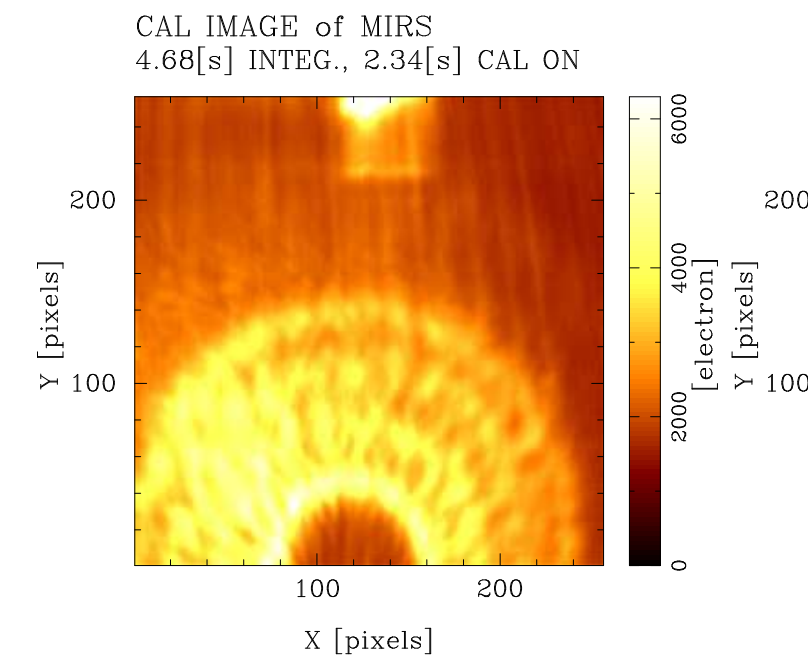

DEAD PIXEL MAP

MIR-S ARRAY

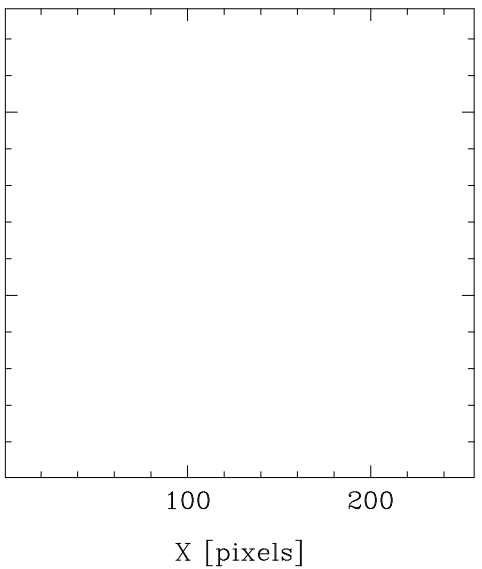

Figure 11. MIR-S image of calibration lamp (left) and bad pixel map (right). Dark (and offset) are subtracted from the lamp image. The pixels whose photo response is less than 1000 electron are shown as dead pixels. Actually, there is NO dead pixel in MIR-S array. 